\title{
TRAWSFFURFIADAU GWYN AP NUDD: O LENYDDIAETH GANOLOESOL I GREDOAU NEO-BAGANAIDD
}

\author{
ANGELIKA HEIKE RÜDIGER
}

Mae nodweddion cymeriadau sy'n cael eu defnyddio mewn rhyddiaith a barddoniaeth am gryn amser wrth gwrs yn newid wrth i'r hanesion gael eu hailadrodd. Bydd y trawsffurfiadau hyn yn amlhau pan fo'r traddodiadau llafar a llenyddol yn effeithio ar ei gilydd, ac mae cymeriad Gwyn ap Nudd (brenin Annwn a brenin y Tylwyth Teg) yn perthyn i'r categori hwn. Mae Gwyn i'w weld yn y traddodiadau rhyddiaith a barddoniaeth o'r canol oesoedd (Bartrum 1993: 351; Foster 1953; Roberts 1980/81; Rüdiger 2012).

Ac yntau wedi ei fabwysiadu gan y mudiad neo-Baganaidd yn hanner cyntaf y ganrif ddiwethaf, caiff ei ddefnyddio mewn credoau sy'n dehongli cymeriadau canoloesol Cymraeg fel hen dduwiau neu dduwiesau (Hutton 1999: 192; Rüdiger 2012: 68-77). Eto, ysgolheigion Cymreig y bedwaredd ganrif ar bymtheg sydd bennaf gyfrifol am ysgogi'r datblygiad hwn sy'n priodoli ystyr newydd i'r cymeriadau. Creffir yn yr ysgrif hon ar y datblygiadau hyn yng nghymeriad Gwyn ap Nudd, o'r testunau hynaf, trwy waith John Rhŷs, hyd Robert Graves a Gerald Gardner a'r credoau neo-Baganaidd.

\section{Gwyn ap Nudd yn yr Oesoedd Canol a'r Cyfnod Modern Cynnar 1.1. Gwyn ap Nudd yn Llyfr Du Caerfyrddin}

Gwelir Gwyn ap Nudd am y tro cyntaf yng ngherdd XXXIV Llyfr Du Caerfyrddin (LIDC: 71-3). Ysgrifennwyd y llawysgrif tua chanol y drydedd ganrif ar ddeg (Huws 2000: 36-56), ond mae iaith y gerdd ymddiddan rhwng Gwyddno Garanhir a Gwyn ap Nudd - yn caniatáu ei dyddio i ddiwedd yr unfed ganrif ar ddeg (Rowland 1990: 388-9). Cyferfydd yr arwyr ar ôl brwydr erchyll yn erbyn llu o Eingl-Sacsoniaid, ac yn dilyn sgwrs o bedwar englyn ar ddeg, ceir saith englyn sy'n rhestru tywysogion ac arwyr yr Hen Ogledd a fu farw ar faes y gad. Mae'n debyg bod y rhan hon yn perthyn i gerdd hynafol, ac yma mae Gwyn yn marwnadu arwyr yr Hen Ogledd. Mae'r ddau englyn olaf yn gosod Gwyn y siaradwr byw mewn cyferbyniad eglur â'r arwyr marw, gan adleisio nodweddion adnabyddus galarnadau'r englynion saga (Rowland 1990). 
Mae'r ymddiddan yn disgrifio Gwyn ap Nudd fel marchog perffaith, rhyfelwr mawr ac 'arbennic llv' (LIDC: 71.2), sef 'arweinydd byddin'. Disgrifir Gwyn gan ddefnyddio ansoddeiriau a nodweddion yr arwr enbyd: mae'n wrol a dewr, yn farchog ardderchog a chanddo farch gwyn (71.19) a chyfrwy euraid (72.28). Mae'n farchog sy'n parchu'r foneddiges Creiddylad. Gallai ei gyflwyno ei hunan fel 'Brenin Annwn' neu 'Pen Annwn', ond yn hytrach dywed, 'hud im gelwir e guin mab nud./ gorterch creurdilad merch lut.' (71.17-18). Mae disgrifiadau fel hyn o arwyr yn weddol gyffredin: fe'u gwelir hefyd yng ngherddi Aneirin, Canu Llywarch Hen a Chanu Heledd. Gelwir Gwyn ap Nudd yn 'darw trin' (71.1), ac mae'r epithet hwn yn y Gododdin, yn disgrifio Eithinyn: 'arderchauc varchawc rac gododin/ eithinyn voleit mur greit tarw trin' (CA: 1lau 431-2), ac yn 'Enweu Meibion Llywarch Hen': 'Tarv trin ryuel adun' (Rowland 1990: 413.14). Mae Trioedd Ynys Prydein yn cynnwys 'Tri Tharw Unben Enys Prydein', a 'Tri Tharw Catuc Enys Prydein' (TYP: 11-13). Disgwylid i arwr fod yn ofnadwy mewn brwydr. Yn y Gododdin disgrifir Madog fel hyn (CA: llau 23-6):

[T]wll tal y rodawr ene klywei

awr. ny rodei nawd mheint dilynei.

ny chilyei o gam-hawn eny verei

weat mal brwyn gomynei gwyr nyt echei.

Mae'r Ymddiddan yn defnyddio geiriau tebyg (LIDC: 72.33-6):

Gwin ab nut but. bitinaur.

kint y sirthei kadoet rac carnetaur dy ueirch.

no bruyn briw y laur.

Mae Gwyn yn mynd ar gefn ceffyl cannaid fel arwyr y Gododdin sy'n mynd ar gefn 'meirch eiliv eleirch' (CA: 1165). Mae eu harfwisgoedd a'u harfau hefyd yn werthfawr a hardd (374-5). Mae'r Ymddiddan yn creu darlun o Gwyn ap Nudd fel y marchog perffaith, gwrol, cywir, sy'n parchu ei foneddiges, ac yn un a allai ymuno'n hawdd â llu enwog y Gododdin, gwrthwynebwyr Lloegr.

Eto, disgrifir Gwyn hefyd fel heliwr goruwchnaturiol nad yw'n aelod o'r gymdeithas ddynol. Mae Gwyn yn sôn am Gaer Vandwy (LIDC: 72.31), sydd hefyd yn y gerdd 'Preiddeu Annwn' (Haycock 2007: 437.42). Hawdd credu y byddai cynulleidfa gyfoes yn ymwybodol o gyddestun Gwyn ap Nudd, brenin Annwn, ac y byddent yn gwybod bod Gwyn yn rhyfelwr pwerus. Yn wir, gallai ei lu achosi diwedd y byd, yn ôl y chwedl Culhwch ac Olwen (CO: 26-7). Felly, y neges amlwg yw nad yw'n bosibl gwrthsefyll yr Eingl-Sacsoniaid: maent yn elyn sy'n medru 
gwrthsefyll nerth goruwchnaturiol y milwr perffaith. Golyga hyn na ddylid gweld arweinwyr y Cymry fel rhyfelwyr gwan, hyd yn oed os na fedrent guro'r gelyn o'r dwyrain. Yn ogystal, mae'r gerdd yn uniaethu brwydro yn erbyn yr Eingl-Sacsoniaid â brwydro yn erbyn tynged ei hun: gellir colli brwydr er gwaethaf ymdrechion milwyr Prydain a llu Annwn. Gwelir bod thema'r gerdd yn addas iawn i englynion chwedlonol, cerddi sydd fel rheol yn archwilio gwrthdrawiadau tyngedfennol (Rowland 1990: 2).

\subsection{Culhwch ac Olwen. Y berthynas rhwng Gwyn ap Nudd a 'Byd yr Herwr'}

Y rhyddiaith gynharaf sy'n sôn am Wyn ap Nudd yw Culhwch ac Olwen, y mae'r copi cynharaf ohono yn Llyfr Gwyn Rhydderch. Ar sail iaith y chwedl, awgrymwyd ar un adeg iddi gael ei hysgrifennu tua 1100 OC (Foster 1953: 199), ond yn fwy diweddar cynigiwyd y drydedd ganrif ar ddeg (Rodway 2005). Yn Culhwch ac Olwen mae Gwyn ap Nudd yn heliwr-ryfelwr a chanddo alluoedd hudol. Mae'r golygyddion diweddaraf yn credu mai cythreuliaid yw trigolion Annwn, deiliaid Gwyn ap Nudd, ac mae Plant Annwn yn sicr yn bygwth hanfodion y byd dynol (CO: 26-7).

Clywir yma hefyd am frwydr enwog Gwyn a Gwythyr dros wrthrych eu serch, Creiddylad: rhaid i Gwyn a Gwythyr frwydro bob Calan Mai hyd Ddydd Brawd (CO: 35-6). Mae'r episod hwn yn cynnwys dau fotiff amlwg o straeon y Tylwyth Teg neu blant Annwn. Mae Calan Mai yn gyfnod pan fo nerth Annwn ar ei gryfaf (Owen 1973: 94-5, 168-9; Rhŷs 2012: 20; Jones 1979: 152-3), a chyffredin mewn chwedlau gwerin yw gweld y Tylwyth Teg yn dawnsio ar nos Galan, pan fo'r drysau i'r byd arall ar agor. Yn ogystal, mae tynged yn gallu newid ar ôl blwyddyn: gellir dwyn pobl yn ôl o'r byd arall ar ôl blwyddyn, a gellir newid canlyniadau gweithredoedd (Owen 1973: 37-40, 46). Mae sawl enghraifft o hyn yng Nghainc Gyntaf y Mabinogi, megis y brwydro rhwng brenhinoedd Annwn, priodas Rhiannon, a dwyn ceffylau a phlant (PKM: 1-27). Ond pwysicaf oll yw'r ffaith mai yn Culhwch ac Olwen y ceir yr eglurhad gorau mewn unrhyw ffynhonnell fod Gwyn yn cyfateb i Fionn mac Cumhaill: mae'r ffynonellau Gwyddelig yn nodi na roddid morwyn mewn priodas heb yn gyntaf ofyn a fyddai un o'r Fianna, llu Fionn, yn deilwng i briodi â hi cyn ei rhoi i ddyn arall (gw. Nagy 1985: 53-4). Mae hyn yn cyfateb i herwgipio Creiddylad. Yn ogystal, yn ôl Murphy (1953: lxiii-lxviii), hen fotiff naratifol sy'n ganolog i'r traddodiad Gwyddelig yw brwydr Fionn yn erbyn cymeriad maleisus a chanddo berthynas â thân. Mae'r motiff storïol hwn, sef brwydro yn erbyn arwr sy'n perthyn i dân, yn ymddangos hefyd yn Culhwch ac Olwen: gelyn Gwyn ap Nudd yw 
Gwythyr ap Greidawl [greidiol < graid 'gwres, llosg']. Gelyn arall i Gwyn yw Greid ap Eri: unwaith eto, wele'r elfen graid.

Yn wir, mae modd gweld Gwyn ap Nudd fel cymeriad a allai berthyn i fyd yr herwr fel y'i disgrifir gan Nagy (1985). Dyna fyd rhyfelwyr-helwyr sy'n byw y tu allan i gymdeithas, yn aros mewn coedwigoedd gwyllt a mynyddoedd anhygyrch. Ar adegau, mewn sefyllfaoedd anodd, bydd y gymdeithas yn gweld angen yr herwyr, ac yn wir, dyma debygrwydd mawr rhwng byd yr herwr Nagy ac Annwn Cymru. Mae Annwn hefyd yn Fyd Arall wedi ei leoli y tu allan i gymdeithas, ac ar adegau gwelir bod angen cymorth Gwyn ap Nudd ar y Brenin Arthur neu ar dywysogion Prydain.

Yn y cyd-destun hwn, gwelir yn episod y gwiddonod fotiff arall sy'n dangos tebygrwydd rhwng Gwyn a Fionn mac Cumhaill. Awgrymir yn Culhwch ac Olwen fod Gwyn a Gwythyr yn hen gyfarwydd â gwiddonod, ac mae'r gwiddonod hynny yn rhyfelwragedd yn hytrach na rheibesau (CO: 41-2). Mae hyn yn gyson â chwedl Peredur, sydd hefyd yn dangos gwiddonod fel rhyfelwragedd (PAE: 35v). Gellir cymharu Gwyn, sy'n deall arferion y gwiddonod, â Fionn mac Cumhaill a gafodd ei addysg gan wragedd yn yr anialdir: rhyfelwragedd a derwyddesau oedd ei athrawesau (Nagy 1985: 99-123).

\subsection{Barddoniaeth Beirdd yr Uchelwyr}

Gwelir yn y gweithiau hyn syniadau sy'n gyson â'r hyn a drafodwyd uchod. Cysylltir Gwyn ap Nudd â natur wyllt, â thir na chaiff ei aredig, ag amserau tywyll a mannau digysur sy'n bell o fyd dynion a'u cymdeithas. Yn gyffredinol, mae'r cerddi sy'n sôn am Gwyn ap Nudd yn rhai chwareus (Roberts 1980/8: 284-5). Mae Dafydd ap Gwilym yn defnyddio cyfeiriadau at Gwyn i ychwanegu lliw i gerddi megis 'Y Niwl', 'Y Pwll Mawn' ac 'Y Dylluan' (DAG 57, 59, 61). Mae Dafydd yn sôn am Gwyn ap Nudd er mwyn disgrifio natur sy'n llesteirio'r bardd rhag cyfarfod â'i gariad ('Y Niwl'), rhag mynd dros y waun heb golli ei ffordd a syrthio i bwll mawn ('Y Pwll Mawn'), neu rhag cysgu ('Y Dylluan'). Y niwl yw 'gwan dalar Gwyn a'i dylwyth' (DAG: 57.40), mae'r pwll mawn yn '[b]ysgodlyn i Wyn' (59.24) a'r dylluan yn 'edn i Wyn ap Nudd' (61.40). Ond mae'r modd y defnyddir enw Gwyn ap Nudd yn chwareus eto. Yn 'Ail Gywydd Ymryson Dafydd ap Gwilym', gŵyr Dafydd fod Gwyn yn gallu herwgipio pobl (DAG 26.54): 'O'm dawr, Gwyn ap Nudd i'm dwyn' ('[...] boed i Wyn ap Nudd fy nghipio'). Yn ogystal, mae Gruffydd Hiraethog yn cysylltu Cŵn Annwn â Gwyn ap Nudd (Roberts 1980/81: 285). Mae hyn yn creu dolen gyswllt â'r heliwr-gythraul sy'n ymddangos 
mewn ofergoelion canoloesol (Lecouteux 2011: 56-60) ${ }^{1}$ ac â'r Helfa Rithiol sy'n syniad mwy modern (Hutton 2014: 175). Mae'n debyg iawn bod dangos Gwyn ap Nudd fel cymeriad sy'n cipio pobl yn dystiolaeth o gyfuno cymeriad traddodiadol lleol â'r trosiad Cristnogol o'r Diafol fel heliwr (gweler 2.4 isod).

\subsection{Speculum Christiani: Tystiolaeth o alw Brenin y Tylwyth Teg}

Mae'r rhan o'r Speculum Christiani a gyfansoddwyd yn y bedwaredd ganrif ar ddeg yn rhoi inni dystiolaeth am arferion gwerin ynghylch Gwyn ap Nudd (Roberts 1980/8: 288). Darllenir yma y byddai pobl yn galw am gymorth Gwyn ap Nudd i wella cleifion a oedd wedi eu melltithio. Mae'r testun yn tystio bod pobl yn lleoli teyrnas Gwyn mewn coedwigoedd dyfnion, ymhell o dir âr, sef lle traddodiadol i gwrdd â bodau Annwn. Mae'r testun yn galw Gwyn ap Nudd yn Frenin yr Eumenides, sef enw ar yr Erinyes neu'r Furiae. Mae'n enw a gaiff ei ddefnyddio i blesio a thawelu'r bodau goruwchnaturiol dialgar hyn (ei ystyr yn llythrennol yw 'duwiesau rhadlon'), yn union fel yr enwau 'Bendith y Mamau' neu 'Tylwyth Teg'. Nid yw'n amhosibl bod y testun yn defnyddio 'Eumenides' i ddisgrifio gwragedd goruwchnaturiol fel gwiddonod neu 'wrachod Annwn' Dafydd ap Gwilym. Yn sicr, mae'n addas iawn defnyddio enw'r Eumenides ar gyfer pobl Gwyn ap Nudd. Mae llên gwerin Cymru yn sôn am ddialgarwch trigolion Annwn: mae straeon Pantannas yn nodweddiadol (Rhŷs 2012: 176-84).

\subsection{Buchedd Collen}

Mae Buchedd Collen (BC), y mae'r fersiwn cynharaf ohoni yn dyddio o 1536, yn dangos Gwyn ap Nudd fel brenin ardderchog ar Annwn, ond mae'r testun yn cynnwys llawer o drosiadau crefyddol sy'n tarddu o draddodiadau sy'n perthyn i waith Beda, Breuddwyd Pawl Ebostol a thadau'r eglwys fel Evagrius Ponticus: er enghraifft, mae gwisg plant Annwn yn arwydd o boenau Uffern, sef gwres ac oerfel (BV lib. iv cap xiii 509 B; BPE). Mae Gwyn ap Nudd yn cwrdd â Chollen am hanner dydd, a'r elfen storïol hon yn uniaethu Gwyn â'r daemonium meridianum

\footnotetext{
1 Yn y drydedd ganrif ar ddeg clywir am infernalis venator ('heliwr-gythraul') mewn testunau gan Caesarius Heisterbach (CH), Jean Gobi (SCG, Rhif 627, tt. 430-31) ac Hélinand o Froidmont (HF, De cognitione sui, cap. XIII, 734 A-735/6 A). Ond mae'n debyg bod y motiff ar led dros Ewrop gyfan. Yn y bedwaredd ganrif ar ddeg mae'n ymddangos yn Alphabetum narrationum (AN, Rhif 464, t.262-63); yn y bymthegfed ganrif yn Summa predicantium mae John Bromyard yn cyfeirio at Hélinand (SPB, XVII,2; f. 91). Mae amrywiad o'r motiff yn ymddangos yn y Decameron hefyd (DEC, tt. 619-27). Mae Lecouteux yn honni bod traddodiad y motiff hwn yn hollol glerigol (Lecouteux 2011: 59). Mae'r heliwr-gythraul yn dwyn pobl neu'u heneidiau. Gwelir bod yr Heliwr Gwyllt yn gymeriad o'r traddodiad gwerin canoloesol sy'n hela cymeriadau goruwchnaturiol eraill.
} 
o athrawiaeth Evagrius Ponticus (di Nola 1993: 224). Mae Buchedd Collen yn ymwneud â bwrw allan ysbrydion, ac ynddo gwelwn fod Gwyn a phlant Annwn yn cael eu hystyried yn gythreuliaid. Mae'r testun yn gosod castell Gwyn ap Nudd ar '[f]ynydd glassymbyri' a uniaethir â Glastonbury Tor (BC: 39), a thystir gan y ddynes hysbys Mary Parish (1631-1703) fod y traddodiad gwerin modern cynnar yn lleoli llys brenhinol y Tylwyth Teg ar Ynys Wydrin (Purkiss 2000: 191). Heddiw mae'r syniad hwn, mai llys Gwyn yw'r Tor, yn bwysig iawn mewn credoau neo-Baganaidd megis Wicca.

I grynhoi: mae Gwyn ap Nudd o destunau'r Oesoedd Canol a dechrau'r cyfnod modern cynnar yn heliwr-ryfelwr, yn farchog perffaith, yn frenin ar blant Annwn (sef cythreuliaid Annwn), ac yn frenin ar ysbrydion dialgar. Mae ei deyrnas wedi ei lleoli y tu allan i'r gymdeithas ddynol a'r byd dynol. Mae'r testunau hyn yn darparu'r deunydd a ddefnyddiwyd gan John Rhŷs, Robert Graves a Gerald Gardner wrth iddynt ddatblygu eu syniadau am Gwyn ap Nudd. ${ }^{2}$

\section{John Rhŷs: Troi Gwyn ap Nudd yn dduw paganaidd 2.1.Gwyn ap Nudd fel Hades Celtaidd}

Yn y bedwaredd ganrif ar bymtheg dechreuwyd rhoi mwy o barch i Astudiaethau Celtaidd a phenodwyd John Rhŷs yn Athro Celtaidd cyntaf Prifysgol Rhydychen. Roedd Rhŷs yn gasglwr llên gwerin brwd. Ffurfiwyd ganddo yn anad neb arall y llun sydd gennym o Gwyn ap Nudd, a ddisgrifiodd fel '[a] repellent personage' (Rhŷs 2008: 560). Credai Rhŷs ei bod yn bosibl darganfod hen dduwiau cyn-Gristnogol y tu ôl i gymeriadau'r chwedlau Arthuraidd. Yn ogystal, gwelir dylanwad mytholeg Glasurol ar ei waith. Roedd methodoleg ei ymchwil i fytholeg Geltaidd yn gymharol, ac roedd mytholeg Roegaidd neu Rufeinig yn sicr

\footnotetext{
${ }^{2}$ Mae'r enw Gwyn ap Nudd yn diflannu o lên gwerin ac o destunau eraill erbyn dechrau'r bedwaredd ganrif ar bymtheg. Yn y cyfnod modern cynnar, mae trigolion Annwn neu'r Tylwyth Teg yn cael eu huniaethu â chythreuliaid, ac mae Annwn yn cael ei droi yn uffern Gristnogol. Mae'r broses hon yn dechrau yn yr Oesoedd Canol, a Culhwch ac Olwen yn rhoi tystiolaeth gynnar ohoni. Mae Gweledigaethau y Bardd Cwsg gan Ellis Wynne yn dangos bod y broses wedi ei chwblhau: Lwsiffer yw tywysog Annwn (GBC: 77) a Belial yw tywysog y Tylwyth Teg (GBC: 11).

Mae Gwyn ap Nudd yn cael ei ail-ddarganfod gan Iolo Morganwg. Creodd Iolo driawd sy'n galw Gwyn ap Nudd yn un o'r tri gwyn seronydd (MA, Tr. 89, trydedd gyfres). Felly, mae Iolo yn disgrifio Gwyn ap Nudd fel derwydd neu ddewin. Yn ei Barddas, mae Iolo Morganwg yn rhoi Gwyn ap Nudd yn safle'r cyntaf o'r doethion (Morganwg 1862-74: 258-9). Ymddangosodd y syniad bod straeon y Tylwyth Teg yn disgrifio hen atgof o'r derwyddon a fu'n byw ynghudd wedi i'r Rhufeiniaid oresgyn Ynys Môn (Cririe 1803: 347; Roberts 1815: 192-201). Felly, naturiol oedd gweld brenin y Tylwyth Teg yn dderwydd mawr. Eto, nid yw hynny'n fawr o ddylanwad ar y syniadau am Gwyn ap Nudd ym maes y ddewiniaeth neo-Baganaidd fodern.
} 
yn cynrychioli rhyw fath o safon iddo. Felly, chwiliai Rhŷs am yr Hades Celtaidd ac mae'n debyg mai hynny, yn ogystal â'i wybodaeth am lên gwerin Gymreig, sy'n gyfrifol am iddo gam-gyfieithu cerdd o Lyfr Du Caerfyrddin.

Cyflawnodd y camgymeriad pwysicaf drwy gyfieithu'r geiriau 'Mi. wi. wiw. vintev. y. bet' (LIDC: 73.60) fel 'I am the escort of the grave' (Rhŷs 2008: 383). Cywirach fyddai 'yr wyf yn fyw, maent hwythau yn y bedd.' Roedd y camgyfieithiad hwn, a'r ffaith fod Gwyn yn dychwelyd o faes y gad, yn ddigon i beri i Rhŷs sôn am Gwyn ap Nudd fel 'god of carnage' (Rhŷs 2008b: 260). Hoffwn gyfeirio'n fyr at ychydig o gamgymeriadau eraill: nid yw Rhŷs yn cydnabod bod Gwyn ap Nudd yn mynd ar gefn ceffyl gwyn. Newidiodd enw'r ci 'Dormach' yn 'Dormarth' a'i gyfieithu fel 'Death's door' (Rhŷs 2008b: 155-6), syniad a wrthodir gan Idris Foster (Foster 1953: 199, 202). Yn ogystal, camddeallodd Rhŷs yr enw 'Gwyn' (Rhŷs 2008a: 84). Ni welai yn y gair ond ystyr y lliw sylfaenol, gan ddiystyru'r cynodiadau 'sanctaidd, pur, etc.', a'r ffaith bod 'Gwyn' yn enw ar arwyr bendigaid a saint. ${ }^{3}$ Galwai Rhŷs Gwyn ap Nudd yn 'god of carnage' neu 'god of the dead', fel petai'n Hades/Plwton Cymreig (Rhŷs 2008: 537), ond nid yw tarddiad y geiriau yn caniatáu hynny.

\subsection{Gwyn ap Nudd fel Brenin y Gaeaf}

Fel y gwelir, aeth Rhŷs yn bellach. Credai fod brwydr Gwyn a Gwythyr yn drosiad o'r gwrthdaro rhwng y tymhorau. Roedd o'r farn bod 'Gwyn yn dwyn Creiddylad' yn cyfateb i 'Plwton yn dwyn Persephone' (mae'n adnabyddus y byddai'r dduwies honno yn aros gyda Plwton (Hades) yn y gaeaf). Am i Gwyn frwydro yn erbyn Gwythyr ap Greidawl, credai Rhŷs mai duw'r haul oedd Gwythyr yn wreiddiol. Yma, dilynai Max Müller, a gredai fod yr hen dduwiau Ariaidd wedi datblygu allan o arferion o addoli nerthoedd natur (Müller 1907). A derbyn bod Gwythyr yn dduw'r haul, uniaethodd Rhŷs y cymeriad hwn â thymor yr haf, ac un unol â hynny ystyriai fod Gwyn ap Nudd yn dduw'r gaeaf a'r tywyllwch. Ond, ar y llaw arall, honnodd Rhŷs fod Fionn mac Cumhaill, sy'n gymeriad cytras â Gwyn ap Nudd, yn dduw'r haul hefyd, fel Gwythyr. Gwelir bod hyn yn peri problem resymegol fawr i safbwynt Rhŷs: mynna fod Fionn mac Cumhaill yn cyfateb i Gwyn ap Nudd ond, yn ôl ei ddehongliad ei hun, mae Gwyn yn gymeriad sy'n gwrthwynebu duw'r haul. Ceisiodd Rhŷs ddatrys y broblem gan uniaethu Gwyn ap Nudd â Finn Eces, 'Fionn arall'

\footnotetext{
${ }^{3}$ Mae Pyll Wyn a Maen Wynn yn Canu Llywarch, 'Pyll' (Rowland 1990: 409, 411); mae Kyndylan wynn yn 'Marwnad Cynddylan' (Rowland 1990: 429), Gwelir 'crist guin' yn LLBC:15.16; a [C]ei guin yn 'Pa ŵr yw'r porthor' (LLBC: 66.3). Am Seiriol Wyn, gw. Mullins (2002: 59).
} 
a chymeriad anfad (Rhŷs 2008a: 560). Ni welai y gallai brwydr Gwyn a Gwythyr gyfateb i frwydrau Fionn mac Cumhaill yn erbyn gelynion tanllyd (gweler uchod 1.2.), ac ni allai dderbyn na fu ond un frwydr y flwyddyn rhwng Gwyn a Gwythyr, oherwydd ei gred mai brwydr rhwng yr haf a'r gaeaf ydoedd honno. Yn wir, honnai fod ail frwydr Gwyn a Gwythyr a ddigwyddasai adeg Calan Gaeaf wedi ei cholli a mynd yn angof. Ni welodd ychwaith y motiff naratifol ei bod yn bosibl dwyn rhywun yn ôl o fyd y Tylwyth Teg ar ôl blwyddyn, sef motiff addas iawn mewn testun sy'n sôn am Gwyn ap Nudd, brenin Annwn a brenin y Tylwyth Teg (Rhŷs 2008a: 559-63).

\subsection{Gwyn ap Nudd fel brenin Gwlad yr Haf}

Cafodd Gwyn ap Nudd ei uniaethu gan Rhŷs â gwahanol gymeriadau a gysylltir â'r Tir Diffaith Arthuraidd, a welai fel teyrnas angau. Rhai enghreifftiau yw Goon of the Desert o barhad y Conte du Graal gan Manessier, a Gornumant, Brenin y Tir Diffaith, gan Gerbert de Montreuil sydd hefyd yn parhau gwaith Chrétien (Rhŷs 2008b: 120-1). Credai Rhŷs hefyd fod Gwyn yn cyfateb i Melwas, Brenin Gwlad yr Haf, ac i gymeriadau tebyg, sef Wanius a Melga, Gunvasius a Malvasius o Historia Regum Britanniae Sieffre o Fynwy, Meleagant o Erec Chrétien de Troyes, a Maheolas o Le Chévalier de la Charette (2008b: 137, 330, 342-4). ${ }^{4}$ Mae Rhŷs yn pwysleisio perthynas Melwas ag Ynys Wydrin. Felly, mae cysylltiad Gwyn â Glastonbury Tor yn gryf iawn, ac mae hyn yn arwain at ddehongliad pellach gan Rhŷs.

\subsection{Gwyn ap Nudd fel Mihangel Sant}

Er y gallai ymddangos yn rhyfedd, tybiai Rhŷs fod Gwyn ap Nudd hefyd yn cyfateb i Mihangel Sant, am fod y ddau yn atal cythreuliaid rhag dinistrio'r byd. Derbyniai Rhŷs y syniad bod y seintiau wedi disodli'r hen dduwiau (Rhŷs 2008b: 341) a chymhara Mihangel Sant sy'n psychopomp â Gwyn ap Nudd, sy'n hela eneidiau. Ond, a bod yn fanwl gywir, nid psychopomp yw Gwyn ap Nudd.

Mae'n debyg bod y darlun o Gwyn ap Nudd fel un sy'n hel eneidiau neu bobl ddrwg yn fotiff a ffurfiwyd drwy gymysgu traddodiadau lleol a chynfrodorol â thraddodiadau Cristnogol yr Oesoedd Canol. Ystyriwn ddwy elfen o'r traddodiadau lleol. Os derbyniwn fod Gwyn yn perthyn i 'fyd yr herwr' ac yn gytras â Fionn mac Cumhaill, gall

\footnotetext{
${ }^{4}$ Gweler yr argraffiadau modern: parhad i'r Conte du Graal gan Manessier: Roach, gol. a Toury, cyf. (2004): parhad Gerbert de Montreuil: Williams \& Oswald, gol. (1922-75); Erec et Enide gan Chrétien de Troyes: Fritz (1992); Le Chevalier de la Charette gan Chrétien de Troyes: Rahilly (1971); Historia Regum Britanniae: Reeve, gol. a Wright, cyf. (2007).
} 
ymddengys yn rhesymol bod Gwyn yn heliwr. Yn ogystal, mae Gwyn a'i bobl yn ddialgar iawn yn llên gwerin Cymru. Yn ôl traddodiad Cristnogol, gall y Diafol fabwysiadau ffurf heliwr: deillia hyn o waith Origen sy'n uniaethu Nimrod, yr heliwr mawr, â'r Diafol oherwydd iddo hefyd wrthod Duw (Herzog, Habermehl a Fuhrmann 2002: 120). Gwelir yn Culhwch ac Olwen fod Annwn yn dechrau cael ei uniaethu ag Uffern: mae trigolion Annwn - sef deiliaid Gwyn - yn gythreuliaid yma. Mae'n nodweddiadol nad yw 'Gwyn yn hela gwŷr drwg' yn fotiff sy'n ymddangos yn echblyg tan farddoniaeth Dafydd ap Gwilym yn y $14 \mathrm{~g}$, ond mae'r motiff 'demoniaid fel helwyr' yn hŷn, ac mae Itinerarium Cambriae Gerallt Gymro yn cynnwys straeon o'r fath, fel straeon Meilyr (IC: 45). Mae hanes Elidorus yn rhan o'r Itinerarium Cambriae hefyd, ac er nad yw Gerallt yn dweud bod unrhyw berthynas rhwng demoniaid Meilyr a phobl bach Elidorus, mae'r bobl bach hyn yn cyfateb i'r Tylwyth Teg (IC: 66-7). Nid yw'n amhosibl uniaethu Gwyn ap Nudd, yr heliwr dialgar cynfrodorol, â'r diafol Cristnogol sy'n hel eneidiau. Eto, anacronistaidd fyddai galw Gwyn ap Nudd yn psychopomp Celtaidd, cyn-Gristnogol sy'n hel eneidiau.

Sylwai John Rhŷs ei hunan nad oedd Gwyn yn y llên gwerin yn mynd ar ôl neb ond gwŷr drwg. Ystyriai fod y motiff sy'n dangos Gwyn fel heliwr eneidiau drwg yn deillio o draddodiad Cristnogol, a honnai y byddai Gwyn yn wreiddiol yn hel eneidiau pawb (Rhŷs 2008b: 155). Gellir cytuno â rhan gyntaf y ddadl hon, ond mae'r ail ran, sef bod Gwyn yn dduw angau sy'n casglu eneidiau - h.y., psychopomp - yn deillio o'r camgyfieithiad a drafodwyd uchod. Gwelir bod dadl Rhŷs yn troi mewn cylch, ar dystiolaeth a grëwyd ganddo ei hunan.

\subsection{Gwyn ap Nudd fel Duw Corniog}

Yn Lectures on the Origin and Growth of Religion as illustrated by Celtic Heathendom, gwnaeth Rhŷs gymhariaeth fawr a arweiniodd at ganlyniadau syncretig pellgyrhaeddol. Credai fod Plwton, duw angau'r Rhufeiniaid, yn gytras â Cernunnos, y duw corniog Rhufeinig-Geltaidd, a bod Cernunnos yn gytras â Heimdall, duw gwyn Aesir. Daliai hefyd fod Heimdall gwyn yn gytras â Gwyn ap Nudd, ac ar sail hyn uniaethai am y tro cyntaf Gwyn ap Nudd a Chernunnos (Rhŷs 2008a: 82-5). Mae'n nodweddiadol fod y gyfres o dduwiau yn dechrau â Plwton, sy'n cyfateb i Hades. Unwaith eto, mae'r ffaith i'r gerdd o Lyfr Du Caerfyrddin gael ei cham-gyfieithu a'i chamddehongli yn arwain at ddeall Gwyn ap Nudd mewn ffordd newydd. Cafodd syniadau Rhŷs eu datblygu a'u rhoi ar led gan Charles Squire (Squire 2003, 254-9). 


\section{Gwyn ap Nudd yn y mudiad neo-Baganaidd \\ 3.1. Robert Graves: Gwyn fel Osiris Cymreig}

Defnyddiai Robert Graves destunau a thraddodiadau Celtaidd hefyd, ac iddo yntau fel ag i eraill, roedd mytholeg Roegaidd, Rufeinig a Dwyreiniol yn fath o ffon fesur safonol a ddylanwadodd ar ei ddehongliadau o'r deunydd Celtaidd. Pwysig iawn iddo oedd gwaith James Frazer am y Duw sy'n marw (Frazer 1894) a syniadau'r Cambridge Ritualists (Doty 2000: 337). Gwaith mwyaf adnabyddus Graves yw The White Goddess (1948), sy'n cynnwys syniadau Graves am fyth barddonol. Erbyn heddiw, mae ffrwyth dychymyg Graves yn seiliau pwysig i syniadau'r Goddess-movement (Hutton 1999: 188-94), mudiad neoBaganaidd sy'n addoli duwies driphlyg (Reid-Bowen 2007: 62-9). Yn ôl monomyth Graves, un thema gyffredin sydd i bob hanes mytholegol, a hynny yw bod dau arwr neu ddau dduw yn cystadlu am dduwies driphlyg mewn brwydr dymhorol, a chredai Graves y byddai gwraig arbennig yn aml yn cymryd lle'r dduwies. Felly, nid yw'r monomyth yn ddim mwy na brwydr rhwng dau ddyn am wraig: mae'n fotiff arbennig o syml, ac yn rhwydd ei ganfod mewn straeon o bob math, yn enwedig os ydys yn chwilio amdano. Dyma'r hyn a wnaeth Robert Graves, wrth gwrs: canolbwyntiodd arno yn ei ddehongliadau, gan anwybyddu bron pob elfen storïol arall.

Mae'r frwydr flynyddol rhyngddo a Gwythyr am Creiddylad yn caniatáu lleoli Gwyn ym monomyth Robert Graves, yn arbennig os caiff y frwydr flynyddol ei throi yn frwydr dymhorol (Graves 1966: 321), yn gyson â syniadau Rhŷs; ond aeth Graves ymhellach: hoff oedd ganddo fodloni'r monomyth gan greu - neu ffugio - elfennau newydd, a lluniodd ddefodau i'w cysylltu â marw a chladdu Gwyn ap Nudd (Graves 1966: 179); cysylltodd Gwyn â'r frwydr dymhorol, a chafodd Gwyn ei droi'n dduw llystyfiant. Yn ôl Robert Graves, Gwyn ap Nudd oedd yr Osiris Cymreig (Graves 1966: 179, 321). Yn ogystal, mae'n cael ei gysylltu â Gwythyr, gan greu duw â dwy agwedd, sef Gwyn a Gwythyr (Graves 1966: 388). Mae Graves yn honni bod un o'r agweddau yn cynnwys triawd o gymeriadau, sef Gwyn, Lleu a Dylan: mae'r ddau arall wrth gwrs yn gymeriadau o Bedwaredd Gainc y Mabinogi. Bwriad ffurfio'r triawd oedd creu cymeriad gwrol a fyddai'n cyfateb i'r dduwies driphlyg ac a fyddai'n debyg i'r Hermes Trismegistos (Graves 1966: 321).

\subsection{Gerald Gardner: Gwyn fel duw cwlt Wicca}

Gerald Gardner yw'r cymeriad enwocaf yn hanes neo-Baganiaeth. Chwaraeai ran ganolog yn natblygiad y ddewiniaeth fodern neoBaganaidd, sef Wicca - yn wir, heb ei ddylanwad yntau, ni fyddai Wicca wedi datblygu ei ffurf bresennol (Hutton 1999: 205-40). Daliai Gardner 
mai Gwyn ap Nudd oedd yr enw amlycaf ar dduw'r gwrachod: coleddai syniadau John Rhŷs a thybiai fod Glastonbury (lleoliad caer Gwyn ym Muchedd Collen) yn ganolfan i addoli'r gwrachod (Gardner 2004: 146). Ond aeth gam ymhellach na Rhŷs a Graves ill dau. Mae Gwyn yn dduw angau i Rhŷs, er mwyn i'r duw tywyll ('the dark divinity') fedru cynnwys bywyd ac angau: felly, mae'r duw tywyll yn dduw ag iddo ddwy agwedd. Ond yn achos Gwyn ap Nudd mae Rhŷs yn dal mai ffurf duw'r lladdfa sydd ar y duw tywyll ac felly nid yw Gwyn yn cynnwys agwedd greadigol y duw tywyll: nid yw'n fwy na 'Hades' (Rhŷs 2008b: 260).

Mae Gardner yn honni hefyd fod Gwyn ap Nudd, sef duw'r gwrachod, yn dduw du sy'n perthyn i angau ac i'r helfa. Mae'n cydnabod bod agwedd greadigol i'r duw du, ond pwysleisia'r agwedd ddinistriol. Hyd yma mae Gardner yn dilyn Rhŷs neu Squire (er nad yw Squire ei hun yn gwneud mwy nag ailadrodd damcaniaethau Rhŷs am Gwyn ap Nudd). Mae Gerald Gardner yn mynd ymhellach gan alw Gwyn ap Nudd yn 'dduw angau ac atgyfodiad', ac yntau'n gymeriad a fyddai'n arwain eneidiau i mewn i'r byd hwn neu i dir ble mae'r meirwon yn aros (Gardner 2004: 145-7, 150). Wrth gymharu Gwyn ap Nudd Gerald Gardner a Gwyn ap Nudd Robert Graves, gwelir bod y ddau yn gysylltiedig ag angau ac atgyfodiad, ond bod y cyntaf yn arglwydd arnynt, a'r olaf yn ddeiliad iddynt.

\section{Casgliadau}

Mae'r ffynonellau cynharaf ar gyfer Gwyn ap Nudd yn ei gyflwyno fel cymeriad sy'n perthyn i 'fyd yr herwr'. Gwelwyd uchod fod Gwyn yn perthyn i gylch sy'n wrthgyferbyniol i gymdeithas ddynol. Yn naturiol, un o nodweddion cymdeithas yw ei chrefydd, ac felly mae Gwyn ap Nudd a Chollen Sant yn cystadlu. Nid yw Gwyn ap Nudd yn gymeriad adeiladol.

Crewyd gan ysgolheigion destunau newydd a ddaeth yn brif ffynonellau i'r mudiadau neo-Baganaidd, ac yn y rhain dim ond y ddelwedd o Gwyn fel heliwr sy'n aros yn gyson: collir bron y cyfan a welir yn yr hen weithiau. Cysylltir syniadau John Rhŷs yn gryf â'r Gwyn newydd: ystyrir bod Gwyn yn hen dduw du, corniog sy'n rheoli tymhorau'r hydref a'r gaeaf, ac fe'i gelwir yn 'Frenin y Gaeaf' gan neoBaganiaid y Deyrnas Unedig, ond yn 'Brenin yr Haf' yn Unol Daleithiau America. Mae'r enw a ddewisir yn dibynnu ar ba rannau penodol o waith John Rhŷs a ddefnyddir (gw. Rüdiger 2012), gan newid y nodweddion sy'n perthyn i Gwyn ap Nudd, a'i statws hefyd.

Mae Gwyn ap Nudd yn newid o fod yn symbol o fyd yr herwr (o Annwn), ac yn ffigwr mewn byd sy'n gyferbyniol i'r byd dynol, i fod yn gymeriad sy'n cynnal y byd dynol hwnnw. Caiff ei droi'n dduw defodol sy'n perthyn i gredoau cwlt megis Wicca. Mae troi Gwyn ap Nudd yn 
dduw cwlt yn troi'r ystyr gwreiddiol sy'n perthyn iddo wyneb i waered am y dylai defodau fod yn hollol absennol o fyd Brenin Annwn. Yn ôl Gerallt Gymro, ni chysylltir y bobl bach y gellir eu huniaethu â'r Tylwyth Teg, deiliaid Gwyn, ag unrhyw gwlt crefyddol, a'u hunig grefydd yw'r gwirionedd: nid ydynt yn tyngu llwon ac nid ydynt yn dweud celwyddau. Dyma yn sicr arferion byd sy'n hollol groes i fyd dynion. Yn y cyd-destun hwn mae'n bosib deall ystyr gwreiddiol Gwyn ap Nudd: mae'n gymeriad goruwchnaturiol sy'n dynodi sffêr wrthwynebol i fyd dynion, ac nid yw'n dduw cwlt.

Serch hynny, dyma un agwedd y dylid ei hystyried. Mae'r neoBaganiaeth sy'n troi Gwyn ap Nudd yn dduw yn seiliedig ar wrthod daliadau Cristnogol prif-ffrwd ein cymdeithas, a hynny, efallai, oherwydd teimlad nad yw'r ffydd Gristnogol yn gallu ateb anghenion ysbrydol ar ôl y Rhyfeloedd Byd. Yn y sefyllfa hon, dewiswyd Gwyn ap Nudd. Yn wreiddiol, bu'n gymeriad a wrthodai gymdeithas, a pherthynai i fyd yr herwr sy'n gallu dod â chymorth mewn argyfwng. Felly, yn reddfol, mae'r mudiadau neo-Baganaidd yn dewis cymeriad sy'n gweddu iddynt ac, yn wir, os dehonglir proses drawsffurfiol Gwyn ap Nudd fel hyn, nid amhosibl ystyried bod ei ystyr gwreiddiol yn fyw o hyd. Mae'r broses o drawsffurfio Gwyn ap Nudd yn parhau bob tro y gwneir defnydd creadigol ohono: mae'n enghraifft ardderchog o'r broses o drawsffurfio cymeriadau ffuglennol neu fytholegol, ac mae'n siŵr nad ef yw'r unig gymeriad y gellid ei astudio yn y modd hwn.

Prifysgol Bangor

\section{Byrfoddau}

AN Brilli, E., gol., 2015, Arnoldi Leodiensis Alphabetum Narrationum, Turnhout.

BC Parry-Williams, T.H. et al., gol., 1954, Rhyddiaith Gymraeg. Y Gyfrol Gyntaf. Detholion o Lawsgrifau (1488-1609), Caerdydd.

BPE James, M.R., 1893, Apocrypha anecdota. A Collection of Thirteen Apocryphal Books and Fragments, Cambridge.

BV Beda Venerabilis, 'In Lucae Evangelium Expositio', yn: Migne, J.-P., gol., 1862, Patrologia Latina, cyf. 92, Paris.

CA Williams, I., gol., 1970, Canu Aneirin, Caerdydd.

CH Caesarius Heisterbach, Dialogus miraculorum, Distictio XII, Cap. XX (https://www.hsaugsburg.de/harsch/Chronologia/ Lspost13-/Caesarius/caedm12.html).

CO Bromwich, R., Evans, D., gol., 1997, Culhwch ac Olwen, Caerdydd. 
DEC Boccaccio, G., Macchi, R., cyf., 2015, Das Dekameron, Darmstadt.

DG Dafydd ap Gwilym.net (http://www.dafyddapgwilym.net/).

GPC Geiriadur Prifysgol Cymru (http://www.geiriadur.ac.uk/).

GBC Donovan, P.J., Thomas, G., gol., 1998, Ellis Wynne. Gweledigaethau y Bardd Cwsg, Llandysul.

HF 'Helinandi Frigidi Montis Monachi necnon Guntheri Cisterciensis Opera Omnia', yn: Migne, J.-P., gol., 1865, Patrologia Latina, cyf. 212, Paris.

IC Gerallt Gymro, gol. R.C. Hoare, D. Powel, 1804, Itinerarium Cambriae, London.

LIDC Jarman, A.O.H., Jones, E.D., gol., 1982, Llyfr Du Caerfyrddin, Gwasg Prifysgol Cymru, Caerdydd.

MA Jones, O., Williams, E. \& Pughe, W.O., 1870, Myvyrian Archaiology of Wales, Denbigh.

PAE Rhyddiaith Gymraeg 1350-1425 (http://www.rhyddiaithganoloe sol.caerdydd.ac.uk/).

PKM Williams, I., gol., 1930, Pedeir Keinc y Mabinogi, Caerdydd.

SCG Polo de Beaulieu, M.-A., gol., 1991, La Scala Coeli de Jean Gobi, Paris.

SPB Johannes Bromiardus, 1409, Summa Predicantium, Nürnberg.

TYP Bromwich, R., gol., 1961, Trioedd Ynys Prydein, Caerdydd.

\section{Llyfryddiaeth}

Bartrum, P.C., 1993, A Welsh Classical Dictionary, Aberystwyth.

Cririe, J., Byrne, W., Walker, G., 1803, Scottish Scenery: or, Sketches in Verse, Descriptive of Scenes Chiefly in the Highlands of Scotland, London.

Denholm-Young, N., 1954, Handwriting in England and Wales, Cardiff. Doty, W.G., 2000, Mythography: The Study of Myths and Rituals, ail arg., Tuscaloosa.

Di Nola, A., 1993, Der Teufel, München.

Foster, I.L., 1953, 'Gwynn ap Nudd', yn: Murphy, G., Duanaire Finn, rhan III, Dublin, 198-204.

Frazer, J.G., 1894, The Golden Bough, London.

Fritz, J.-M., gol., 1992, Chrétien de Troyes, Erec et Enide, Paris.

Gardner, G., 2004, The Meaning of Witchcraft, York Beach, ME.

Graves, R., 1966, The White Goddess, New York.

Haycock, M., 2007, Legendary Poems from the Book of Taliesin, Aberystwyth. 
Herzog, R., Habermehl, P., Fuhrmann, M., 2002, Spätantike: Studien zur römischen und lateinisch-christlichen Literatur, Göttingen.

Huws, D., 2000, Medieval Welsh Manuscripts, Cardiff.

Hutton, R., 1999, The Triumph of the Moon: A History of Modern Pagan Witchcraft, Oxford.

Hutton, R., 2014, 'The Wild Hunt and the Witches' Sabbath', Folklore $125,161-78$.

Jarman, A.O.H., 1985, 'Llyfr Du Caerfyrddin - The Black Book of Carmarthen. Sir John Rhŷs Memorial Lecture', Proceedings of the British Academy LXXI, 333-56.

Jarman, A.O.H., Jones, E.D., gol., 1982, Llyfr Du Caerfyrddin, Caerdydd.

Jones, T.G., 1979, Welsh Folklore and Folk-Custom, Cambridge (2 ${ }^{\text {il }}$ argraff).

Lecouteux, C., 2011, Phantom Armies of the Night, Inner Traditions, Rochester, Vermont.

Morganwg, I., 1862-74, The Barddas of Iolo Morganwg, cyfr. I, gol. J. Williams ab Ithel, Llandovery, London.

Müller, F.M., 1907 [1888], Natural Religion: The Gifford Lectures Delivered before the University of Glasgow, London.

Mullins, D.J., 2002, Seintiau Cynnar Cymru, Llanrwst.

Murphy, G., 1953. Duanaire Finn: The Book of the Lays of Fionn. Part III, Dublin.

Nagy, J. F., 1985, The Wisdom of the Outlaw, Berkley.

Owen, E., 1973, Welsh Folk-Lore, Norwood (adargraffiad ffacsimili o argraffiad 1896).

Purkiss, D., 2000, At the Bottom of the Garden: A Dark History of Fairies, Hobgoblins, and Other Troublesome Things, New York.

Rahilly, L. J., 1971, The Garrett Manuscript No 125 of Chrétien's 'Chevalier de la Charette' and 'Chevalier au Lion': A Critical Edition, Princeton.

Reid-Bowen, P., 2007, Goddess as Nature: Towards a Philosophical Thealogy, Aldershot, T.U.

Rhŷs, J., 2008a, Lectures on the Origin and Growth of Religion as illustrated by Celtic Heathendom, London (adargraffiad ffacsimili o argraffiad 1888).

Rhŷs, J., 2008b, Studies in Arthurian Legend, Oxford (adargraffiad ffacsimili o argraffiad 1891 gan Kessinger: www.kessinger.net).

Rhŷs, J., 2012, Celtic Folklore Welsh and Manx, Oxford (adargraffiad ffacsimili o argraffiad 1901 gan Forgotten Books: www.forgotten.books.org). 
Roach, W., gol., Toury, M.-N., cyf., Manessier, La Troisème Continuation du Conte de Graal, 2004, Paris.

Roberts, B.F., 1980/81, 'Gwyn ap Nudd', Llên Cymru, XIII, 283-9.

Roberts, P., 1815, The Cambrian Popular Antiquities (Collectanea Cambrica), London.

Rodway, S., 2005, 'The Date and Authorship of Culhwch ac Olwen: a Reassessment', Cambrian Medieval Celtic Studies, 49, 21-44.

Rowland, J., 1990, Early Welsh Saga Poetry, Cambridge.

Rüdiger, A.H., 2012, 'Gwyn ap Nudd. Transfigurations of a Character on the Way from Medieval Literature to Neo-Pagan Beliefs', Traethawd M.A. anghyhoeddedig, Prifysgol Bangor.

Squire, C., 2003, Celtic Myth and Legend, Poetry and Romance, Holicong PA.

Williams, I., gol., 1930, Pedeir Keinc y Mabinogi allan o Lyfr Gwyn Rhydderch, Caerdydd.

Williams, M., Oswald, M., gol., 1922-75, Classiques Français du Moyen Age: Gerbert de Montreuil, La Continuation de Perceval, Paris. 